\title{
EDUCAÇÃO EM SAÚDE: percepção de profissionais atuantes em uma Coordenadoria Regional de Saúde
}

\author{
Roger Flores CECCONa, Kelly de M oura OLIVEIRA, \\ M icheli Scolari ROSSET T Oc, A lessandra Regina M üller GERM AN I ${ }^{d}$
}

\section{RESUMO}

0 objetivo do estudo é analisar a percepção dos profissionais atuantes em uma Coordenadoria Regional de Saúde sobre a E ducação em Saúde. T rata-se de uma pesquisa qualitativa do tipo exploratório-descritiva. Foi realizada uma entrevista com oito profissionais do setor público estadual, responsáveis pela implementação das políticas públicas de saúde. Para análise foi usado o método análise de conteúdo do tipo temática. Os resultados foram discutidos com base nas propostas de Paulo $\mathrm{F}$ reire. O bservou-se que os profissionais de saúde detêm percepções diferenciadas no que tange questões que per meiam a educação e educação em saúde, tendo suas práticas educacionais pautadas no sistema tradicional. Assim, é necessária uma mudança neste contexto, unificando conceitos e construindo uma nova práxis educacional, fazendo rever as práticas a todo o momento e incorporando novos saberes pautados na construção de um novo modelo de saúde.

D escritores: E ducação em saúde. Pessoal de saúde. Percepção.

\section{RESUMEN}

El objetivo es analizar las percepciones de los profesionales que trabajan en Salud R egional deE ducación en Salud. E se trata de una investigación cualitativa exploratorio-descriptiva. F ueron realizadas entrevistas con ocho profesionales del sector público estatal, encargados de ejecutar las políticas públicas de salud. Para el análisis se utilizó el método de análisis de contenido temático. L os resultados fuer on discutidos con baseen las propuestas de $P$ aul o $F$ reire $F$ ue señalado que los profesi onales de la salud tienen percepciones diferentes sobre cuestiones relacionadas con la educación y la educación en la salud, y sus prácticas educativas sopor tadas por el sistema tradicional. E sto requi er e un cambio en este contexto, la unificación de conceptos y la construcción de una nueva praxis educativa, haciendo una reseña de las prácticas en todo momento y la incorporación de nuevos conocimientos guiado la construcción de un nuevo modelo de salud.

Descriptores: E ducación en salud. Personal de salud. P ercepción.

T ítulo: E ducación en salud: percepciones de profesionales actuantes en un Coordinadora R egional de Salud.

\section{ABST RACT}

Theaim of this study is to analyz etheperceptions of professionals working in a R egional $\mathrm{H}$ ealth $\mathrm{O}$ fficein $\mathrm{H}$ ealth $\mathrm{E}$ ducation. T his is a qualitative exploratory-descriptive study. Interviews w ere held with eight professionals from the state public sector, responsi blefor implementing public health policies. For anal ysis w e used anal yzing thematic content methodology. The results w erediscussed based on proposals of Paulo $\mathrm{F}$ reire It was noted that health professionals havedifferent perceptions regarding questions related to education and health education, and their educational practices are supported by the traditional system. The situation calls for a change, which includes unifying concepts and buil ding a new educational praxis, reviewing practices at all times and incorporating new knowledge to guide the construction of a new model of health.

Descriptors: $\mathrm{H}$ ealth education. $\mathrm{H}$ ealth personnel. P erception.

T itle: $\mathrm{H}$ ealth education: perceptions of professionals w orking in a R egional $\mathrm{H}$ ealth Office

\footnotetext{
a M estrando pelo Programa de Pós-G raduação em Enfermagem da U niversidade Federal do Rio G rande do Sul (UFRGS), Professor dos Cursos T écnicos do Departamento de Ciências da Saúde da U niversidade de Cruz Alta (U N I CRU Z), Cruz Alta, Rio G rande do Sul, Brasil. - M estranda pelo Programa de Pós-G raduação em Saúde Coletiva da U niversidade Luterana do Brasil (U LBRA), Professora do Curso de Enfermagem do Departamento de Ciências da Saúde da U NICRUZ, Cruz Alta, Rio G rande do Sul, Brasil.

M estranda pelo Programa de Pós-G raduação em Saúde Coletiva da U LBRA, Porto Alegre, Rio G rande do Sul, Brasil.

${ }^{d} M$ estre em Enfermagem, Professora do D epartamento de Enfermagem da U niversidade Federal Fronteira Sul, Porto Alegre, Rio G rande do Sul, Brasil.
} 


\section{INT RODUÇÃO}

A construção de um sistema de saúde democrático, universal, igualitário e integral, constituise em um processo amplo, social e político, que se concretiza por meio da implementação de políticas públicas voltadas para a realidade de saúde apresentada pela população, e também pela problematização do cotidiano de assistência prestada nos serviços de saúde ${ }^{(1)}$.

A educação em saúde está ancorada em práticas de promoção da saúde, que trata de processos que abrangem a participação de toda a população e não apenas das pessoas sob risco de adoecer. Essa noção está baseada em um conceito de saúde, considerado como um estado positivo e dinâmico de busca de bem-estar, que integra os aspectos físicos e mentais, ambiental, pessoal e social ${ }^{(2)}$.

0 ato de educar não pode ser apenas uma forma de depositar, narrar, transferir ou de transmitir conhecimentos e valores aos educandos, mas sim ser um ato cognoscente, cedendo lugar para uma educação problematizadora, que sugere a superação da contradição educador-educandos ${ }^{(3)}$. A ssim, esta relação dialógica se torna possível quando 0 pensamento crítico, inquieto, e que neste caso, do profissional da saúde, não impede na capacidade de refletir do usuário. 0 verdadeiro diálogo precisa ser construído entre sujeitos mediatizados pela realidade(4).

Quanto aos desafios contemporâneos da educação e promoção de saúde, estes não podem afrontar-se exclusivamente a partir do exercício de delegação nos sistemas profissionais de tipo sanitário, com seus sofisticados protocolos para a investigação, intervenção e informação(5).

A educação em saúde favorece a emancipação do sujeito e promove 0 autocuidado. Ressalta-se, com isso, a importância de construir uma consciência crítica, que leve os sujeitos a pensar sobre a for mação de suas identidades ${ }^{(6)}$. Paralelo a isso, também, é preciso oferecer aos profissionais que proporcionam espaços de educação uma formação adequada, possibilitando condições e segurança na prática educativa(7).

A tualmente, há diferentes movimentos articulando-se ao mesmo tempo, ou seja, ainda permanece a educação tradicional, centrando o poder nas mãos do profissional de saúde, e a educação popular, que no início era considerada como método al ter nativo de prática educativa. A educação po- pular em saúde sai da margem da sociedade e incorpora outras práticas e espaços educativos, na busca do empoderamento por parte da comunidade, baseando-se no encorajamento e apoio, para que as pessoas e grupos sociais assumam maior controle sobre sua saúde e suas vidas ${ }^{(8)}$.

A educação em saúde precisa ser compreendida como uma proposta que desenvolve no indivíduo e no grupo a capacidade de analisar de forma crítica a sua realidade, decidir ações conjuntas para resolver problemas e modificar situações vigentes ${ }^{(9)}$. A ssim, o diálogo, a troca de conhecimentos, os questionamentos e a participação popular representam uma importante abertura para transformações positivas ${ }^{(10)}$.

Em suma, a prática de educação em saúde instrumentaliza indivíduos e grupos para se autoorganizarem a desenvolverem ações a partir de suas próprias prioridades, orienta e estimula à participação dos sujeitos nas ações dirigidas à melhoria de suas condições de vida e saúde ${ }^{(11)}$.

A eficiência no processo educativo depende, dentre outras coisas, da capacidade do educador em entender a leitura do mundo feita pelo educando e, a partir dessa leitura, ampliar o seu conhecimento, levando o educando a ter uma visão mais crítica. N esse sentido, partir do saber do outro não significa ficar preso a ele, mas considerar que seu conteúdo possa servir de base para melhor conhecer o sujeito ${ }^{(12)}$.

U ma das formas práticas para obtenção de êxito no processo educativo é a inserção do educador na comunidade, o conhecimento dos mitos e tabus locais sobre determinados temas e 0 envolvimento com lideranças locais, muitas vezes representadas pelos agentes comunitários de saúde ${ }^{(13)}$.

A proposta surgiu a partir das diferentes realidades no desenvolvimento de políticas públicas que norteiam a educação em saúde, tendo em vista a existência de diferentes métodos e conceitos que norteiam a prática educativa, aliada a necessidade de desenvolver atividades de educação em saúde para sujeitos protagonistas.

A partir de tal situação, torna-se pertinente e necessário entender as percepções dos profissionais diante da temática educação em saúde, considerando a relevância do tema na prática profissional, principalmente aos sujeitos cujo papel está centrado na formulação, execução e avaliação de políticas públicas de saúde, tendo em vista o desenvolvimento de ações de promoção em saúde em 
consonância com práticas educacionais pautadas em um modelo resolutivo.

0 objetivo do estudo é analisar a percepção de profissionais atuantes em uma Coordenadoria Regional de Saúde sobre a E ducação em Saúde.

\section{MET ODOLOGIA}

T rata-se de uma pesquisa qualitativa do tipo exploratório-descritiva, inserida na linha de pesquisa "Promoção, Prevenção e Reabilitação" da U niversidade Regional Integrada do A Ito U ruguai e M issões.

Todas as etapas do estudo decorreram entre os meses de junho de 2006 a maio de 2007, tendo como propósito analisar as percepções de profissionais, atuantes em uma Coordenadoria Regional de Saúde, responsáveis pela formulação, execução e avaliação de políticas públicas sobre educação em saúde no estado do Rio G rande do Sul.

A coleta de dados foi realizada em oito momentos distintos, entre os meses de outubro a dezembro de 2006, em horários alternados, nos espaços de atuação dos profissionais, em sede própria da Coordenadoria Regional de Saúde. F oi realizada entrevista semi-estruturada, com questões abertas e fechadas, utilizando instrumento de coleta de dados baseado em questionamentos que fomentaram saberes e percepções relacionadas às temáticas "educação", "educação em saúde", "métodos educacionais" e "sujeitos participantes das práticas educativas".

Foram incluídos os profissionais de ensino superior responsáveis pelas políticas públicas de saúde e excluídos os profissionais com ensino superior incompleto e/ ou que não eram responsáveis pela implementação de políticas públicas de saúde nos municípios de abrangência.

Foram selecionados oito profissionais que atenderam os critérios de inclusão, sendo esclarecidos os objetivos e procedimentos da pesquisa. Os participantes assinaram um Termo de Consentimento Livre e Esclarecido.

Para tratamento do material, utilizou-se a técnica de análise de conteúdo do tipo análise temática, sendo organizado e estruturado seguindo as fases sequenciais: pré-análise, exploração do material e tratamento dos resultados ${ }^{(14)}$. Os resultados foram descritos por categorizações, expondo os achados encontrados na análise.
Os dados foram discutidos com base nas propostas de Paulo $\mathrm{F}$ reire, a qual nos levou a reflexões a cerca dos temas presentes nas falas dos sujeitos da pesquisa, fazendo rever as práticas a todo o momento e incorporando novos saberes pautados na construção de um novo modelo de saúde ${ }^{(3)}$.

A pesquisa respeitou a Resolução 196/ 96 do Consel ho Nacional de Saúde ${ }^{(15)}$ e foi aprovada pelo Comitê de Ética e Pesquisa da U niversidade Regional I ntegrada do A to U ruguai e das M issões (URI), sob o no 0127.0.284.000-06.

\section{RESULTADOS E DISCUSSÃO}

Os resultados do presente estudo, identificados nos depoimentos, foram dispostos a partir de significados e percepções dos sujeitos da pesquisa e discutidos com ênfase em conceitos que nor teiam a educação e práticas educativas em saúde ${ }^{(16)}$.

U m dos grandes desafios da educação, para trabalhar com as ideias de Paulo F reire, é produzir o diálogo liber tador a partir de uma educação problematizadora, focando o diálogo, a humanização da assistência, os vínculos, o acolhimento nos serviços de saúde e a participação, não usando o diálogo autoritário, denominada de educação bancária(4).

As temáticas identificadas nas entrevistas, refer entes ao questionamento proposto aos profissionais sobre suas percepções de educação em saúde, foram agrupadas e classificadas em três categorias: conceituando educação em saúde (1), métodos educacionais (2) e sujeitos participantes das práticas educacionais (3).

\section{Conceituando E ducação em Saúde}

O conteúdo presente nessa categoria referese à conceituação, percepção e importância da educação em saúde na prática profissional, o que fica evidenciado nos discursos do participante 7 e do participante 4, respectivamente.

São os saber es transmitidos às pessoas em sua vida através do conhecimento, da aprendizagem, compreensão e vivência do dia-a-dia (Participante 7).

A to de passar ensinamentos as pessoas (Participante 4).

E ssas falas, referentes à educação em saúde, podem ser atribuídas a um conjunto de saberes e 
práticas orientadas para a prevenção de doenças e promoção da saúde. T rata-se de um recurso por meio do qual o conhecimento cientificamente produzido no campo da saúde, intermediado pelos profissionais, contribui para a formação de autonomia, uma vez que a compreensão dos condicionantes do processo saúde-doença oferece subsídios para a adoção de novos hábitos de saúde(1).

Com isso, a proposta de educação ultrapassa os limites de uma teoria, porquanto ela pode ser entendida como forma de compreender o mundo, refletir sobre ele e transformar a realidade a partir de uma ação consciente ${ }^{(16)}$.

$\mathrm{N}$ a escola emancipatória o educando é o sujeito da história, com ele o educador é capaz de trocar infor mações acerca do mundo que o rodeia, fazendo com que este sujeito tenha um ol har crítico sobre a realidade na qual ele está inserido, tornando-o capaz de atuar na sociedade de uma forma dinâmica, criativa, coletiva, tendo princípios éticos e políticos ${ }^{(17)}$.

E ducação é um processo contínuo de aprendizagem, onde o sujeito faz sucessivas aproximaçōes com 0 objeto, sempre causando uma mudança, um olhar crítico sobrea realidade (Participante 3 ).

0 participante citado conceitua educação de uma forma abrangente, entendendo a educação como um processo de mudanças, tendo o sujeito como participante do processo educativo.

É a partir do saber fundamental da mudança de um mundo mais justo e igualitário que programamos nossa ação político-pedagógica, não importando se o projeto com o qual nos comprometemos é de al fabetização de adultos ou de crianças, se de ação sanitária, se de evangelização, se de formação de mão-de-obra técnica, é necessário a conscientização de que a mudança é possível| ${ }^{(17)}$.

\section{Métodos E ducacionais}

N essa categoria, os participantes abordam os métodos e as formas que são utilizadas para a realização das práticas educacionais, o que fica enfatizado na colocação a seguir:

É a forma de passar conhecimentos sobre educação e saúde e ensinar as pessoas a usá-los de forma clara e concisa (Participante 5).

É a capacidade de transpor conhecimentos, informações, legitimar saberes (Participante 8).
Percebe-se, através das falas, que é presente, no cotidiano prático, o modelo de transmissão de conteúdos, conhecido como "educação bancária", inserido no modelo tradicional de educação, associado aos bancos da escola, onde o professor deposita no aluno o seu conhecimento. A avaliação no modelo tradicional é um processo sistemático contínuo e integral, avaliando se o conhecimento foi transmitido com sucesso e se os objetivos educacionais foram al cançados ${ }^{(17)}$.

Assim, podemos observar que o modelo tradicional de educação em saúde está fortemente enraizado nas práticas educativas realizadas pelos profissionais de saúde. N este modelo, a transmissão do conhecimento técnico-científico é privilegiada, sendo o educador o detentor do saber e 0 educando um depósito a ser preenchido pelo educador $^{(18)}$.

N esse sentido, não existe educação sem liberdade de criar e de propor o quê e como aprender, herdando a experiência adquirida, criando e recriando, integrando-se às condições de seu contexto, respondendo os seus desafios, objetivandose a si próprio, discernindo, transcendendo, Iançando-se no domínio da história e o da cultura ${ }^{(5)}$.

A prática cotidiana da educação em saúde está intimamente relacionada à educação depositária e vertical, contrapondo-se a uma ótica mais ampla que atende as complexidades da nova saúde pública. Esta propõe uma abordagem que busca fortalecer a consciência crítica das pessoas, para uma participação ativa no delineamento de suas circunstâncias de vida ${ }^{(9)}$.

A educação pode e deve ser muito mais que um processo de treinamento ou domesticação, um processo que nasce da observação e da reflexão e culmina na ação transfor madora(16).

0 educando deve ser reconhecido como sujeito portador de um saber sobre o processo saúdedoença-cuidado, sendo capaz de estabelecer uma interlocução dialógica com o serviço de saúde e de desenvolver uma análise crítica sobre a realidade e 0 aperfeiçoamento das estratégias de enfrentamento e luta ${ }^{(19)}$.

No modelo dialógico de educação em saúde, o indivíduo é reconhecido sujeito portador de um saber, que embora distinto do saber técnico-científico não é deslegitimado pelos serviços. $\mathrm{E} m$ um modelo dialógico e participativo, todos, profissionais e usuários, atuam como iguais, ainda que com papéis diferenciados ${ }^{(20)}$. 
Percebe-se tentativas de mudança no âmbito da metodologia de educação em saúde pelos profissionais atuantes.

E ducação em Saúdeé saber mos ou conhecer mos o meio em que vivemos e atuamos, em todo o seu contexto e saber mos tomar medidas viáveis para a promoção da saúde individual e coletiva (Participante 6).

E ducação em saúde para mim refere se como uma das atividades dos profissionais desaúde, e porquecada indivíduo ér esponsável em promover, prevenir, reabilitar sua condição de saúde visando, ser um agente de mudança (Participante 1).

Os participantes enfatizam que, para a realização de educação em saúde, deve-se primeiramente ser conhecida a realidade, para posterior mente elaborar um planejamento de ações de promoção de saúde de qualidade.

A educação problematizadora permite a troca de conhecimento e, consequentemente, a construção dele a partir das experiências vivenciadas, favorecendo a metodologia quando bem explorada. A ssociado a isso, o processo de ensinagem é aplicado auxiliando nessa construção(21).

Assim, reforça-se com o discurso do participante 7:

São as diversas maneiras de aprendizagem através de experiências vividas com finalidade de melhorar a saúde da população (Participante 7).

Foi evidenciado no estudo que uma parte das práticas de educação nos serviços de saúde está voltada para a superação do fosso cultural existente entre a instituição e os indivíduos, em que um lado não compreende a lógica e as atitudes do outro, nessas experiências, isso é feito com base em uma perspectiva de compromisso com os interesses políticos das classes populares, mas reconhecendo-Ihe, cada vez mais, a diversidade e a heterogeneidade ${ }^{(5)}$.

\section{Sujeitos Participantes das Práticas E ducacionais}

$N$ essa categoria foram apresentadas as percepções dos profissionais no que se refere à participação nas práticas educacionais, bem como os sujeitos envolvidos no processo educativo, o que é apresentado na fala do participante 8:
A educação é uma das atividades dos profissionais de saúde, sendo cada indivíduo responsável em promover, prevenir e reabilitar sua condição de saúde visando ser um agente de mudança (Participante 8).

Assim, a conscientização individual sobre a importância da educação em saúde é um compromisso histórico, é uma inserção crítica na história, assumindo o homem uma posição de sujeito, podendo transformar o mundo. É o desenvolvimento crítico da tomada de consciência. É um ir além da fase espontânea da apreensão até chegar a uma fase crítica na qual a realidade se torna um objeto cognoscível e se assume uma posição epistemológica procurando conhecer é tomar posse da realidade; e, por esta razão, e por causa da radicação utópica que a informa, é um afastamento da realidade ${ }^{(5)}$.

0 principal promotor de saúde éo indivíduo, e por isso não deve se centralizar ações somente nos profissionais de saúde, mas sim envolver cada entidade, cada cidadão, cada profissional disposto a participar deste processo saúde doença (Participante 3 ).

0 participante 5 enfatiza os sujeitos participantes da educação em saúde na prática diária da E stratégia Saúde da F amília:

T rabalhamos na C oordenação da E stratégia Saúdeda F amília e, através dela, com ações educacionais, quere mos tornar o cidadão mais autônomo, transmitindo informações e favorecendo a essas pessoas viver de forma saudável, sabendo escolher o que é mel hor para sua saúde ea fazer uso de todos os seus direitos sem esque cer de seus deveres (Participante 5).

A possibilidade de troca conjunta de saberes entre equipe de saúde e usuários do serviço é uma visão teoricamente moderna, apresentando-se como uma prática inovadora e na realidade ainda ínfima. E sta prática se depara com barreiras culturais muito arraigadas, o que impossibilita a abertura necessária para a inserção do cidadão/ usuário como sujeito ativo no processo educativo ${ }^{(9)}$.

A incorporação da educação em saúde às práticas da estratégia de saúde da família se mostra cada vez mais atual e necessária, principalmente quando esta ocorre a partir da troca de conhecimentos, estabelecendo mais do que um ensino e uma aprendizagem um ato de criar e transfor$\operatorname{mar}^{(9)}$. 


\section{CONCLUSÕES}

A educação proposta por F reire ultrapassa os limites do "achismo", de "acomodação", de "fracassos", ela vai além porque obtém princípios de igualdade, de trocas de saberes, de convivência e de círculo de cultura.

Os profissionais estudados são educadores que tiveram sua educação pautada pela pedagogia do modelo tradicional, porém incorporando tentativas de construção de saberes e práticas educacionais, tendo como uma das formas de efetivação a Estratégia Saúde da Família.

Os profissionais possuem diferentes percepções do conceito prático-conceitual no que tange a educação e educação em saúde, sendo estes, individualmente, responsáveis por políticas educacionais no contexto em que atuam.

É necessário promover o debate na construção col etiva dos profissionais do que significa educação em saúde, seus conceitos e da importância do uso no cotidiano profissional para incorporar o processo educacional, permeando que todos são atores sociais e educadores em potencial.

Quanto mais se articula o conhecimento frente ao mundo, mais os educandos se sentirão desafiados a buscar respostas, e consequentemente quanto mais incitados, mais serão levados a um estado de consciência crítica e transformador a frente à realidade. E sta relação dialética precisa ser incorporada na medida em que, educadores e educandos se fazem sujeitos do seu processo.

\section{REFERÊNCIAS}

1 Alves VS. U m modelo de educação em saúde para o Programa Saúde da F amília: pela integralidade da atenção e reorientação do modelo assistencial. Interface Comun Saúde E duc. 2004/ 2005;9(16):39-52.

2 M achado M FAS, M onteiro EM LM, Queiroz DT, Vieira N F C, Barroso M G T. I nteg ralidade, formação de saúde, educação em saúde e as propostas do SU S: uma revisão conceitual. Ciênc Saúde Colet. 2007;12 (2):335-42.

3 F reire P. Pedagogia do oprimido. 49a ed. Rio de Janeiro: Paz e Terra; 2007.

4 Heidemann IBS, Boehs AE, Wosny A M, Stulp KP. Incorporação teórico-conceitual e metodológica do educador Paulo F reire na pesquisa. Rev Bras E nferm. 2010;63(3):416-20.
5 M artínez-H ernáez A. Dialógica, etnografia e educação em saúde. Rev Saúde Pública. 2010;44(3):399405.

6 Freitas KR, Dias SM Z. Percepções de adolescentes sobre sua sexualidade. T exto Contexto E nferm. 2010; 19(2):351-7.

7 Caldeira AP, A guiar GN , M agal hães WAC, F agundes $G C$. Conhecimentos e práticas de promoção do aleitamento materno em E quipes de Saúde da F amília em M ontes Claros, M inas G erais, Brasil. Cad Saúde Pública. 2007;23(8):1965-70.

8 Silva CM C, M eneghin M C, Pereira AC, M ialhe FL. E ducação em saúde: uma reflexão histórica de suas práticas. Ciênc Saúde Colet. 2010;15(5):2539-50.

9 Santos AS. Educação em saúde: reflexão e aplicabilidade em atenção primária á saúde. Online Braz J N urs [ I nter net] . 2006 [ citado 2007 set 26] ;5(2). Disponível em: http:/ / www.objnursing.uff.br// index.php/ nursing/ article/ view/ 435.

10 F ernandez M CP, Backes V M S. E ducação em saúde: perspectivas de uma equipe da E stratégia Saúde da Família sob a óptica de Paulo Freire. Rev Bras Enferm. 2010;63(4):567-73.

11 Colomé JS, Oliveira D L LC. A educação em saúde na perspectivas de graduandos de enfermagem. Rev G aúcha E nferm. 2008;29(3):347-53.

12 Vasconcelos M LM C, Brito RH P. Conceitos de educação em Paulo F reire: glossário. Petrópolis: Vozes; São Paulo: M ack Pesquisa; 2006.

13 Parada CM GL, Carval haes M ABL, W inckler CC, W inckler $L A, W$ inckler VC. Situação do al eitamento materno em população assistida pelo programa de saúde da família-PSF. Rev Latino-Am E nfermagem. 2005;13(3):407-14.

14 M inayo M CS. Pesquisa social: teoria, método e criatividade. Petrópolis: Vozes; 2001.

15 M inistério $\mathrm{N}$ acional da Saúde (BR), Conselho N acional de Saúde. Resolução 196/ 96 sobre pesquisa envolvendo seres humanos. Bioética 1996;4(2):15-25.

16 F reire $P$. E ducação e mudanças. 30 a ed. Rio de Janeiro: Paz e T erra; 2007.

17 F reire P. Pedagogia da autonomia: saberes necessários à prática educativa. São Paulo: Paz eT erra; 1997. 
18 Figueiredo M F S, Rodrigues-N eto JF, Leite M T S. $M$ odel os aplicados às atividades de educação em saúde. Rev Bras Enferm. 2010;63(1):117-21.

19 T oledo M M, Rodrigues SC, Chiesa AM . E ducação em saúde no enfrentamento da hipertensão arterial: uma nova ótica para um velho problema. Texto Contexto Enferm. 2007;16(2):233-8.
20 Alves VS. A health education model for the Family $\mathrm{H}$ ealth Program: towards comprehensive heal th care and model reorientation. Interface Comun Saúde E duc. 2005;9(16):39-52.

21 Santos VOG, Vargens OM C. A prática discente na construção do conhecimento sobre o fenômeno das drogas. Rev G aúcha Enferm. 2010;31(1):41-7.
Endereço do autor / Dirección del autor / Author's address:

Roger F lores Ceccon

Rua E I pídio G oulart, 101, Rocha

98025-280, Cruz Alta, RS

E-mail: roger.ceccon@ hotmail.com
Recebido em: 26/ 09/2010

A provado em: 18/ 01/ 2011 